\title{
Numbers with fixed sum of digits in linear recurrent number systems
}

\author{
Stephan G. Wagner*
}

\begin{abstract}
We study the set of integers with a given sum of digits with respect to a linear recurrent digit system. An asymptotic formula for the number of integers $\leq N$ with given sum of digits is determined, and the distribution in residue classes is investigated, thus generalizing results due to Mauduit and Sárközy. It turns out that numbers with fixed sum of digits are uniformly distributed in residue classes under some very general conditions. Namely, the underlying linear recurring sequence must have the property that there is no prime factor $P$ of the modulus such that all but finitely many members of the sequence leave the same residue modulo $P$. The key step in the proof is an estimate for exponential sums using known theorems from Diophantine approximation.
\end{abstract}

MSC 2000 Classification: 11A63,11K65

Key words: Linear recurrent digit system, sum of digits, residue distribution

\section{Introduction and Notation}

Linear recurrent digit systems are a generalization of the usual radix representations; they have been studied, for example, in $[3,12,14,15,21]$. We start with a definition of these systems:

Let $G=\left(G_{n}\right)(n=0,1, \ldots)$ be a linear recurring sequence of order $d \geq 1$, i.e.

$$
G_{n+d}=a_{1} G_{n+d-1}+a_{2} G_{n+d-2}+\ldots+a_{d} G_{n}
$$

with integral coefficients and integral initial values. We assume that the coefficients $a_{1} \geq a_{2} \geq \ldots \geq a_{d}>$ 0 are non-increasing $\left(a_{1}>1\right.$ if $\left.d=1\right)$ and that $G_{0}=1$ and

$$
G_{n}>a_{1}\left(G_{0}+\ldots+G_{n-1}\right), n=1, \ldots, d-1 .
$$

For an arbitrary positive integer $N$, we define $L=L(N)$ by $G_{L} \leq N<G_{L+1}$ (and set $L(0)=0$ ). Furthermore, set $N_{L}=N$,

$$
\epsilon_{j}=\left\lfloor\frac{N_{j}}{G_{j}}\right\rfloor, N_{j-1}=N_{j}-G_{j} \epsilon_{j}(1 \leq j \leq L),
$$

and finally $\epsilon_{0}=N_{0}$, yielding a unique representation of $N$ of the form

$$
N=\sum_{j=0}^{L(N)} \epsilon_{j} G_{j},
$$

the $G$-ary representation of $N$ with digits $\epsilon_{j}$. If $d=1$ and $a_{1}=g$, we obtain the well-known base- $g$ representation of $N$.

*Affiliation of author: Department of Mathematics, Graz University of Technology, Steyrergasse 30, A-8010 Graz, Austria; email: wagner@finanz.math.tu-graz.ac.at 
Now, the sum of digits is naturally defined as

$$
s_{G}(N)=\sum_{j=0}^{L(N)} \epsilon_{j}
$$

The best-known instance of such a digit system is probably the Zeckendorf expansion [22], belonging to the Fibonacci sequence $G_{0}=1, G_{1}=2, G_{n+2}=G_{n+1}+G_{n}$.

In [21], Pethő and Tichy generalized a well-known result of Delange [5] on the mean value of the sum of digits to linear recurring sequences. For usual base- $g$ expansions, numbers with fixed sum of digits were studied by Mauduit and Sárközy in [19]. Their first main result states that the number of integers with $\leq \nu$ digits and sum of digits $k \leq \frac{g-1}{2} \nu$ (for reasons of symmetry, this case is obviously sufficient) is, uniformly for $k \rightarrow \infty$,

$$
r^{-k}\left(1+r+\ldots r^{g-1}\right)^{\nu} \pi^{1 / 2}(D \nu)^{-1 / 2}\left(1+O(D \nu)^{-1 / 2}\right)
$$

where the implied constant depends only on the base $g ; r$ is defined as the unique positive zero of

$$
Q(x)=-k\left(1+x+\ldots+x^{g-1}\right)+\nu x\left(1+2 x+\ldots+(g-1) x^{g-2}\right),
$$

and $D=2 \pi^{2}\left(B-A^{2}\right)$, where

$$
A=\left(\sum_{j=1}^{g-1} j r^{j}\right)\left(\sum_{j=0}^{g-1} r^{j}\right)^{-1}=\frac{k}{\nu} \text { and } B=\left(\sum_{j=1}^{g-1} j^{2} r^{j}\right)\left(\sum_{j=0}^{g-1} r^{j}\right)^{-1} .
$$

Secondly, they showed that the integers with fixed sum of digits are uniformly distributed in residue classes if the modulus is not too large and relatively prime to $(g-1) g$ - this theorem was further generalized in a very recent paper of Mauduit, Pomerance and Sárközy [17], relaxing the condition that the modulus is relatively prime to $(g-1) g$. Furthermore, they were able to prove an Erdős-Kac-type theorem for integers with fixed sum of digits.

Similar results for other kinds of digitally restricted sets are due to Erdős, Mauduit and Sárközy ([8, 9], integers with missing digits), Fouvry and Mauduit resp. Mauduit and Sárközy $([10,11,18]$, integers with congruence conditions for the sum of digits).

In this paper, we are going to prove a generalization of formula (3) to linear recurrent digit systems and study the distribution in residue classes. It turns out that we have uniform distribution if there is no prime divisor $P$ of the modulus such that $\left(G_{n}\right)$ is constant modulo $P$ for all but finitely many values of $n$.

We will make use of the following notational conventions: we write $e(\alpha)=\exp (2 \pi i \alpha)$, we use $c_{1}(G), c_{2}(G), \ldots$ for constants which depend only on the basis $G$ of our digital system, and we write $f(N)=O_{G}(g(N))$, if there is a constant $C(G)$ depending only on $G$ such that, for sufficiently large $N, f(N) \leq C(G) g(N)$ holds.

\section{Asymptotic enumeration}

We start with a characterization of admissible digital expansions given by Pethő and Tichy in [21]:

Lemma 1 The $(t+1)$-tuple $\left(\epsilon_{0}, \ldots, \epsilon_{t}\right) \in \mathbb{N}_{0}^{t+1}$ is the sequence of $G$-ary digits of an integer if and only if

$$
\sum_{j=0}^{n} \epsilon_{j} G_{j}<G_{n+1}
$$


for all $0 \leq n<d-1$ and

$$
\left(\epsilon_{n}, \ldots, \epsilon_{n-d+1}\right)<\left(a_{1}, \ldots, a_{d}\right)
$$

lexicographically (i.e. there is an $i$ such that $\epsilon_{n+1-j}=a_{j}$ for $j<i$ and $\epsilon_{n+1-i}<a_{i}$ ) for all $d-1 \leq n \leq t$.

This lemma enables us to establish a generating function for the integers with fixed sum of digits:

Proposition 2 Let $F(k, \nu)$ be the set of integers with $\leq \nu$ base- $G$ digits and sum of digits $k$. Then we have

$$
|F(k, \nu)|=\left[x^{\nu} y^{k}\right] \frac{p(x, y)}{q(x, y)},
$$

where $p(x, y)$ and $q(x, y)$ are polynomials and $q(x, y)$ is given by

$$
q(x, y)=1-\sum_{i=1}^{d}\left(\sum_{j=0}^{a_{i}-1} y^{j}\right)\left(\prod_{l=1}^{i-1} y^{a_{l}}\right) x^{i} .
$$

Proof. By the preceding lemma, we have to consider sequences satisfying the two conditions (4) and (5). We call such sequences good. Let a good sequence $\left(\epsilon_{0}, \ldots, \epsilon_{t}\right)$ be given. By (5), there is an $i$ such that $\epsilon_{t+1-j}=a_{j}$ for $j<i$ and $\epsilon_{t+1-i}<a_{i}$. The remaining digits $\left(\epsilon_{0}, \ldots, \epsilon_{t-i}\right)$ obviously form a good sequence. Conversely, a sequence $\left(b, a_{i-1}, \ldots, a_{1}\right)$ with $b<a_{i}$ may be appended to any good sequence of length $\geq d$ to form another good sequence. Thus, if

$$
g(t)=\sum_{\epsilon} y^{s(\epsilon)}
$$

where the sum is over all good sequences $\epsilon=\left(\epsilon_{0}, \ldots, \epsilon_{t}\right)$ and $s(\epsilon)=\epsilon_{0}+\ldots+\epsilon_{t}$, we have

$$
g(t)=\sum_{i=1}^{d}\left(\sum_{j=0}^{a_{i}-1} y^{j}\right)\left(\prod_{l=1}^{i-1} y^{a_{l}}\right) g(t-i)
$$

if $t$ is large enough. This shows that the generating function for our problem is given by a rational function of the form $\frac{p(x, y)}{q(x, y)}$, with $q(x, y)$ as in (6).

Lemma 3 Let $q(x, y)$ be given by (6), and define $\lambda=\lambda(y)$ for positive $y$ as the unique positive solution to $q(\lambda, y)=0$. Furthermore, define

$$
\mu(y)=-\frac{y \lambda^{\prime}(y)}{\lambda(y)}=\frac{y q_{y}(\lambda(y), y)}{\lambda(y) q_{x}(\lambda(y), y)} .
$$

Then $\mu(y)$ is a continuous, strictly increasing function with $\lim _{y \rightarrow 0} \mu(y)=0$ and $\lim _{y \rightarrow \infty} \mu(y)=A=$ $\max _{i} \frac{a_{1}+\ldots+a_{i}-1}{i}$. Furthermore, there exists a constant $c_{1}(G)>0$ depending on $G$ such that $\mu^{\prime}(y) \geq c_{1}(G)$ for all $y \in[0,1]$.

Proof. Obviously, $q(x, y)$ is strictly decreasing in $x$ and $y$, and $q(0, y)=1$, whereas $q(x, y) \rightarrow-\infty$ as $x \rightarrow \infty$. Therefore, $\lambda(y)$ is well-defined, and so is $\mu(y)$. Clearly, $\lambda(y)$ and $\mu(y)$ are continuous. As $q(x, 0)=1-x$, we know that $\lambda(0)=1$. Furthermore, $q_{x}(x, 0)=-1$, which means that $\mu(0)=0$.

Since $\lambda(y)$ is an algebraic function with no branch points on $[0, \infty)$ (note that the derivative $q_{x}(\lambda(y), y)$ is strictly negative on this interval), $\lambda(y)$ has a holomorphic continuation and is thus infinitely often differentiable. Since $\lambda(y) \neq 0$ for all $y$, this also holds for $\mu(y)$.

$r(x, y)=1-q(x, y)$ is a polynomial in $x, y$ with positive coefficients and constant coefficient 0 . We write $r(x, y)=\sum_{k, l} r_{k l} x^{k} y^{l}$. Implicit differentiation yields

$$
\mu(y)=\frac{y q_{y}(\lambda(y), y)}{\lambda(y) q_{x}(\lambda(y), y)}=\frac{y r_{y}(\lambda(y), y)}{\lambda(y) r_{x}(\lambda(y), y)}
$$


and

$$
\begin{aligned}
\mu^{\prime}(y)= & \frac{1}{x^{3} y r_{x}(x, y)^{3}}\left(y^{2} r_{y}(x, y)^{2}\left(x r_{x}(x, y)+x^{2} r_{x x}(x, y)\right)+x^{2} r_{x}(x, y)^{2}\left(y r_{y}(x, y)+y^{2} r_{y y}(x, y)^{2}\right)\right. \\
& \left.-2 x^{2} y^{2} r_{x}(x, y) r_{y}(x, y) r_{x y}(x, y)\right)\left.\right|_{x=\lambda(y)} .
\end{aligned}
$$

The denominator is positive for $y>0$. The numerator can be written as

$$
\begin{aligned}
& \left(\sum_{k, l} l r_{k l} x^{k} y^{l}\right)^{2}\left(\sum_{k, l} k^{2} r_{k l} x^{k} y^{l}\right)+\left(\sum_{k, l} k r_{k l} x^{k} y^{l}\right)^{2}\left(\sum_{k, l} l^{2} r_{k l} x^{k} y^{l}\right) \\
& -2\left(\sum_{k, l} k r_{k l} x^{k} y^{l}\right)\left(\sum_{k, l} l r_{k l} x^{k} y^{l}\right)\left(\sum_{k, l} k l r_{k l} x^{k} y^{l}\right) .
\end{aligned}
$$

We set $u_{k l}=\sqrt{r_{k l} x^{k} y^{l}}, v_{k l}=k \sqrt{r_{k l} x^{k} y^{l}}$ and $w_{k l}=l \sqrt{r_{k l} x^{k} y^{l}}$. Then this equals

$$
\begin{gathered}
\left(\sum_{k, l} u_{k l} w_{k l}\right)^{2}\left(\sum_{k, l} v_{k l}^{2}\right)+\left(\sum_{k, l} u_{k l} v_{k l}\right)^{2}\left(\sum_{k, l} w_{k l}^{2}\right)-2\left(\sum_{k, l} u_{k l} v_{k l}\right)\left(\sum_{k, l} u_{k l} w_{k l}\right)\left(\sum_{k, l} v_{k l} w_{k l}\right) \\
=\langle\mathbf{u}, \mathbf{w}\rangle^{2}\langle\mathbf{v}, \mathbf{v}\rangle+\langle\mathbf{u}, \mathbf{v}\rangle^{2}\langle\mathbf{w}, \mathbf{w}\rangle-2\langle\mathbf{u}, \mathbf{v}\rangle\langle\mathbf{u}, \mathbf{w}\rangle\langle\mathbf{v}, \mathbf{w}\rangle,
\end{gathered}
$$

where $\langle.,$.$\rangle denotes the scalar product. Combining the inequality between the arithmetic and geometric$ mean and the Cauchy-Schwarz inequality yields

$$
\begin{aligned}
\langle\mathbf{u}, \mathbf{w}\rangle^{2}\langle\mathbf{v}, \mathbf{v}\rangle+ & \langle\mathbf{u}, \mathbf{v}\rangle^{2}\langle\mathbf{w}, \mathbf{w}\rangle-2\langle\mathbf{u}, \mathbf{v}\rangle\langle\mathbf{u}, \mathbf{w}\rangle\langle\mathbf{v}, \mathbf{w}\rangle \\
& \geq 2 \sqrt{\langle\mathbf{u}, \mathbf{w}\rangle^{2}\langle\mathbf{v}, \mathbf{v}\rangle\langle\mathbf{u}, \mathbf{v}\rangle^{2}\langle\mathbf{w}, \mathbf{w}\rangle}-2\langle\mathbf{u}, \mathbf{v}\rangle\langle\mathbf{u}, \mathbf{w}\rangle\langle\mathbf{v}, \mathbf{w}\rangle \\
& \geq 2 \sqrt{\langle\mathbf{u}, \mathbf{w}\rangle^{2}\langle\mathbf{u}, \mathbf{v}\rangle^{2}\langle\mathbf{v}, \mathbf{w}\rangle^{2}}-2\langle\mathbf{u}, \mathbf{v}\rangle\langle\mathbf{u}, \mathbf{w}\rangle\langle\mathbf{v}, \mathbf{w}\rangle \\
& =0
\end{aligned}
$$

with equality if and only if $\mathbf{v}, \mathbf{w}$ are linearly dependent. In our case, this can only be if $r_{k l} \neq 0$ happens only for one value of $\frac{k}{l}$. By our conditions on the $a_{i}$, this is impossible. Therefore, $\mu^{\prime}(y)>0$ for all $y \in(0, \infty)$, which implies that $\mu(y)$ is strictly increasing. Direct calculation shows that $\mu^{\prime}(0)=1$. So $\mu^{\prime}(y)$ is continuous and positive on the compact interval $[0,1]$ and has thus a minimum $c_{1}(G)>0$.

Finally, we note that $r(x, y)$ behaves like

$$
\sum_{i} y^{\sum_{l=1}^{i} a_{l}-1} x^{i}
$$

for $y \rightarrow \infty$. Now it is easy to see that

$$
q_{y}(\lambda(y), y) \sim \sum_{i} \frac{i A}{y} \text { and } q_{x}(\lambda(y), y) \sim \sum_{i} \frac{i}{\lambda(y)},
$$

where the sum is over all $i$ (there might be more than one) for which $\frac{a_{1}+\ldots+a_{i}-1}{i}=A$. It follows immediately that $\lim _{y \rightarrow \infty} \mu(y)=A$.

REMARK. It is easily proved that $A=a_{1}-\frac{1}{M} \geq \frac{1}{2}$, where $M$ is the largest index such that $a_{1}=a_{M}$.

Lemma 4 Let $\lambda_{1}(y)$ be the solution of smallest modulus of $q(x, y)=0$ for arbitrary complex $y$, and let $\lambda_{2}(y)$ be one of the solutions of second-smallest modulus. Then there exist constants $\phi(G), c_{2}(G), c_{3}(G), \kappa_{1}(G)$ depending only on the sequence $G$ such that $c_{2}(G)<1, \kappa_{1}(G)>0$ and

$$
\left|\frac{\lambda_{1}(y)}{\lambda_{2}(y)}\right| \leq \min \left(c_{2}(G), c_{3}(G)|y|^{\kappa_{1}(G)}\right)
$$

for all $y \in B=\{z \in \mathbb{C}:|z| \leq 1,|\arg z| \leq \phi(G)\}$ and $\lambda_{1}$ coincides with the branch $\lambda$ on $B$. 
Proof. $\lambda_{1}(y)$ coincides with $\lambda(y)$ on the compact interval $[0,1]$, since we already know that $\lambda(y)$ is the unique solution of minimal modulus on this interval. Note that all branches of the equation $q(x, y)=0$ except $\lambda$ tend to $\infty$ with some negative power of $y$ as $y \rightarrow 0$. Therefore, there exists some $\delta>0$ such that $\lambda_{1}(y)=\lambda(y)$ and

$$
\left|\frac{\lambda_{1}(y)}{\lambda_{2}(y)}\right| \leq c_{4}(G)|y|^{\kappa_{1}(G)}
$$

for all $y$ with $|y| \leq \delta$, where $c_{4}(G), \kappa_{1}(G)$ are constants depending on $G$.

The absolute distance to the second-smallest solution is a continuous function on $(0,1]$, and it tends to $\infty$ as $y \rightarrow 0$, so it has a minimum on $[0,1]$.

Furthermore, if we choose $\epsilon_{1}$ small enough to avoid all the (finitely many) branch points of the equation $q(x, y)=0$ - there are none on $[\delta / 2,1]-$, all branches are holomorphic on $[\delta / 2,1] \times\left[-\epsilon_{1}, \epsilon_{1}\right]$, so they satisfy a Lipschitz condition. This means that we can find $\epsilon_{2}>0$ such that $\lambda$ is the unique branch of smallest modulus on $[\delta / 2,1] \times\left[-\epsilon_{2}, \epsilon_{2}\right]$.

Choose $\phi(G)$ small enough such that $B$ is contained in

$$
\{y \in \mathbb{C}:|y| \leq \delta\} \cup[\delta / 2,1] \times\left[-\epsilon_{2}, \epsilon_{2}\right] .
$$

$B$ is a compact set, and the function $f(y)=\left|\frac{\lambda_{1}(y)}{\lambda_{2}(y)}\right|$ is continuous on this set, if we take $f(0)=0$. Thus it has a maximum, which must be $<1$. Take this as the constant $c_{2}(G)$. Then, $(9)$ holds for some constant $c_{3}(G)$.

\section{Corollary 5}

$$
f(x, y)=\frac{p(x, y)}{q(x, y)}-\frac{p\left(\lambda_{1}(y), y\right)}{q_{x}\left(\lambda_{1}(y), y\right)\left(x-\lambda_{1}(y)\right)}
$$

is a holomorphic function on $\left\{x \in \mathbb{C}:|x|<\left|\lambda_{2}(y)\right|\right\}$ for all $y \in B$, and there exist constants $c_{5}(G), \kappa_{2}(G)$ depending only on $G$ such that

$$
|f(x, y)| \leq c_{5}(G) y^{-\kappa_{2}(G)}
$$

holds on $\left\{x \in \mathbb{C}:|x| \leq \sqrt{\left|\lambda_{1}(y)\right|\left|\lambda_{2}(y)\right|}\right\}$. As a consequence,

$$
\left[x^{\nu}\right] \frac{p(x, y)}{q(x, y)}=-\frac{p\left(\lambda_{1}(y), y\right)}{q_{x}\left(\lambda_{1}(y), y\right)} \lambda_{1}(y)^{-\nu-1}\left(1+O_{G}\left(\eta_{G}^{-\nu}\right)\right),
$$

where $\eta_{G}>1$ depends only on $G$.

Proof. Note that $\frac{p\left(\lambda_{1}(y), y\right)}{q_{x}\left(\lambda_{1}(y), y\right)\left(x-\lambda_{1}(y)\right)}$ is the principal part of $\frac{p(x, y)}{q(x, y)}$ at $x=\lambda_{1}(y)$, so $f(x, y)$ is indeed holomorphic, since $\frac{p(x, y)}{q(x, y)}$ has a single pole at $\lambda_{1}(y)$ and no other singularity for $|x|<\left|\lambda_{2}(y)\right|$. Now, we write

$$
q(x, y)=r(y)\left(x-\lambda_{1}(y)\right)\left(x-\lambda_{2}(y)\right) \ldots\left(x-\lambda_{d}(y)\right)
$$

for $y \in B \backslash\{0\}$ and note that

$$
q_{x}\left(\lambda_{1}(y), y\right)=r(y)\left(x-\lambda_{2}(y)\right) \ldots\left(x-\lambda_{d}(y)\right),
$$

yielding

$$
f(x, y)=\frac{p(x, y)}{r(y)\left(x-\lambda_{1}(y)\right)}\left(\frac{1}{\left(x-\lambda_{2}(y)\right) \ldots\left(x-\lambda_{d}(y)\right)}-\frac{1}{\left(\lambda_{1}(y)-\lambda_{2}(y)\right) \ldots\left(\lambda_{1}(y)-\lambda_{d}(y)\right)}\right) .
$$

$y$ is bounded on $B$, and $|x|<\left|\lambda_{2}(y)\right|$ can be bounded by a power of $y$. Furthermore, the factors $\left(x-\lambda_{i}(y)\right)$ are bounded below by $\left|\lambda_{2}(y)\right|\left|1-\sqrt{\left|\frac{\lambda_{1}(y)}{\lambda_{2}(y)}\right|}\right|$ for $x \leq \sqrt{\left|\lambda_{1}(y)\right|\left|\lambda_{2}(y)\right|}$, and the factors $\left(\lambda_{1}(y)-\lambda_{i}(y)\right)$ by $\left|\lambda_{2}(y)\right||1-| \frac{\lambda_{1}(y)}{\lambda_{2}(y)}||$. 
Altogether, we see that (10) holds for some constant $c_{5}(G)$ if $y \in B \backslash\{0\}$ and $|x| \leq \sqrt{\left|\lambda_{1}(y)\right|\left|\lambda_{2}(y)\right|}$. For $y=0$, however, the claim is essentially trivial.

Now, we have

$$
\left[x^{\nu}\right] \frac{p(x, y)}{q(x, y)}=\left[x^{\nu}\right] \frac{p(x, y)}{q_{x}\left(\lambda_{1}(y), y\right)\left(x-\lambda_{1}(y)\right)}+\left[x^{\nu}\right] f(x, y)
$$

and

$$
\left[x^{\nu}\right] f(x, y)=\oint_{\mathcal{C}} x^{-\nu-1} f(x, y) d x \leq 2 \pi c_{5}(G) y^{-\kappa_{2}(G)}{\sqrt{\left|\lambda_{1}(y)\right|\left|\lambda_{2}(y)\right|}}^{-\nu}
$$

where $\mathcal{C}$ is the circle of radius $\sqrt{\left|\lambda_{1}(y)\right|\left|\lambda_{2}(y)\right|}$ around 0. Finally,

$$
\left[x^{\nu}\right] \frac{p(x, y)}{q_{x}\left(\lambda_{1}(y), y\right)\left(x-\lambda_{1}(y)\right)}=-\frac{p\left(\lambda_{1}(y), y\right)}{q_{x}\left(\lambda_{1}(y), y\right)} \lambda_{1}(y)^{-\nu-1}
$$

for $\nu>\operatorname{deg}_{x} p(x, y)$. The claim now follows from the preceding lemma.

Next, we need a lemma from [19]:

Lemma 6 (Mauduit/Sárközy [19]) For $g>1,0<r \leq 1$ and all $\alpha \in \mathbb{R}$ we have

$$
\left|\frac{1+r e(\alpha)+r e(2 \alpha)+\ldots+r^{g-1} e((g-1) \alpha)}{1+r+r^{2}+\ldots+r^{g-1}}\right| \leq 1-\frac{2 r}{g}\|\alpha\|^{2} .
$$

Lemma 7 There exist constants $c_{6}(G), c_{7}(G)$ depending only on $G$ such that

$$
\left|\left[x^{-\nu}\right] \frac{p(x, r e(\alpha))}{q(x, r e(\alpha))}\right| \leq c_{6}(G) \exp \left(-c_{7}(G) r \nu\|\alpha\|^{2}\right)\left[x^{\nu}\right] \frac{p(x, r)}{q(x, r)}
$$

for all $0<r \leq 1$ and all $\alpha \in \mathbb{R}$.

Proof. Note that $z_{\nu}(y):=\left[x^{\nu}\right] \frac{p(x, y)}{q(x, y)}$ is a polynomial with positive coefficients in $y$. So, obviously, $z_{\nu}($ re $(\alpha)) \leq z_{\nu}(r)$ for all $\nu$. Furthermore, $z_{\nu}(y)$ satisfies a recurrence relation of the form

$$
z_{\nu}(y)=\sum_{i=1}^{d}\left(\sum_{j=0}^{a_{i}-1} y^{j}\right)\left(\prod_{l=1}^{i-1} y^{a_{l}}\right) z_{\nu-i}(y)
$$

It follows that

$$
\left|z_{\nu}(y)\right| \leq \sum_{i=1}^{d}\left|\sum_{j=0}^{a_{i}-1} y^{j} \prod_{l=1}^{i-1} y^{a_{l}}\right|\left|z_{\nu-i}(y)\right|
$$

First, we assume that $a_{1}>1$. Then, by the previous lemma,

$$
\left|\sum_{j=0}^{a_{1}-1}(r e(\alpha))^{j}\right| \leq\left(1-\frac{2 r}{a_{1}}\|\alpha\|^{2}\right) \sum_{j=0}^{a_{1}-1} r^{j}
$$

Trivially,

$$
\left|\sum_{j=0}^{a_{i}-1}(r e(\alpha))^{j}\left(\prod_{l=1}^{i-1}(r e(\alpha))^{a_{l}}\right)\right| \leq \sum_{j=0}^{a_{i}-1} r^{j} \prod_{l=1}^{i-1} r^{a_{l}}
$$

for all $i>1$. Now, if we define $Z_{\nu}(r, \alpha)$ by $Z_{\nu}(r, \alpha)=z_{\nu}(r)$ for $\nu<d$ and

$$
Z_{\nu}(r, \alpha)=\left(1-\frac{2 r}{a_{1}}\|\alpha\|^{2}\right) \sum_{j=0}^{a_{1}-1} r^{j} Z_{\nu-1}(r, \alpha)+\sum_{i=2}^{d} \sum_{j=0}^{a_{i}-1} r^{j} \prod_{l=1}^{i-1} r^{a_{l}} Z_{\nu-i}(r, \alpha),
$$


we know that $Z_{\nu}(r, \alpha) \geq\left|z_{\nu}(r e(\alpha))\right|$ for all $\nu$. Since

$$
\left(1-\frac{2 r}{a_{1}}\|\alpha\|^{2}\right) \sum_{j=0}^{a_{1}-1} r^{j} \geq\left(1-\frac{r}{4}\right)(1+r)=1+\frac{r(3-r)}{4} \geq 1,
$$

$Z_{\nu}(r, \alpha)$ is an increasing sequence. Furthermore,

$$
\sum_{j=0}^{a_{1}-1} r^{j} \geq \sum_{j=0}^{a_{i}-1} r^{j} \prod_{l=1}^{i-1} r^{a_{l}}
$$

for all $i \geq 2$, since $r \leq 1$ and the $a_{i}$ are decreasing. It follows that

$$
\begin{aligned}
Z_{\nu}(r, \alpha) & \leq\left(1-\frac{2 r}{a_{1} d}\|\alpha\|^{2}\right) \sum_{i=1}^{d} \sum_{j=0}^{a_{i}-1} r^{j} \prod_{l=1}^{i-1} r^{a_{l}} Z_{\nu-i}(r, \alpha) \\
& \leq \exp \left(-\frac{2 r}{a_{1} d}\|\alpha\|^{2}\right) \sum_{i=1}^{d} \sum_{j=0}^{a_{i}-1} r^{j} \prod_{l=1}^{i-1} r^{a_{l}} Z_{\nu-i}(r, \alpha) \\
& \leq \sum_{i=1}^{d} \exp \left(-\frac{2 r i}{a_{1} d^{2}}\|\alpha\|^{2}\right) \sum_{j=0}^{a_{i}-1} r^{j} \prod_{l=1}^{i-1} r^{a_{l}} Z_{\nu-i}(r, \alpha)
\end{aligned}
$$

and thus

$$
Z_{\nu}(r, \alpha) \leq c_{6}(G) \exp \left(-c_{7}(G) r \nu\|\alpha\|^{2}\right) z_{\nu}(r)
$$

for constants $c_{6}(G), c_{7}(G)=\frac{2}{a_{1} d^{2}}$ by simple induction on $\nu$. This proves the claim in the case of $a_{1}>1$. If $a_{1}=a_{2}=\ldots=a_{d}=1$, iterate the recurrence equation for $z_{\nu}$ once to obtain

$$
z_{\nu}(y)=\sum_{i=2}^{d} y^{i-2}(1+y) z_{\nu-i}(y)+y^{d-1} z_{\nu-d-1}
$$

and apply the same method to this equation (note that we have at least one term of the form $(1+y)$, as $d \geq 2$ in this case).

Now, we are ready to prove our first main theorem following the same line of proof as Mauduit and Sárközy:

Theorem 8 Let $F(k, \nu)$ be defined as in Proposition 2 and take $A$ as in Lemma 3. Then, uniformly for $l=\min (k, A \nu-k) \rightarrow \infty$, we have

$$
|F(k, \nu)|=\frac{p(\lambda(r), r)}{-\lambda(r) q_{x}(\lambda(r), r)} \pi^{1 / 2}(D \nu)^{-1 / 2} r^{-k} \lambda(r)^{-\nu}\left(1+O_{G}\left((D \nu)^{-1 / 2}\right)\right),
$$

where $r$ is defined by $\mu(r)=\frac{k}{\nu}$ and $D=2 \pi^{2} r \mu^{\prime}(r)$.

Proof. From Proposition 2, we know that

$$
|F(k, \nu)|=\left[x^{\nu} y^{k}\right] \frac{p(x, y)}{q(x, y)} .
$$

First, let $\frac{k}{\nu} \leq \mu(1)$. Choose $0<r \leq 1$ in such a way that $\mu(r)=\frac{k}{\nu}-$ this is possible by Lemma 3 . Now, we have

$$
|F(k, \nu)|=r^{-k} \int_{-1 / 2}^{1 / 2}\left[x^{\nu}\right] \frac{p(x, r e(\alpha))}{q(x, r e(\alpha))} e(-k \alpha) d \alpha .
$$

We split the integral in two parts: define

$$
J_{1}=\int_{-\delta}^{\delta}\left[x^{\nu}\right] \frac{p(x, r e(\alpha))}{q(x, r e(\alpha))} e(-k \alpha) d \alpha
$$


and

$$
J_{2}=\int_{\delta<|\alpha| \leq 1 / 2}\left[x^{\nu}\right] \frac{p(x, r e(\alpha))}{q(x, r e(\alpha))} e(-k \alpha) d \alpha,
$$

where $\delta=k^{-1 / 2} \log k$. We will deal with $J_{1}$ first. If $k$ is large enough, we have $\delta<\phi(G)$, so we may apply Corollary 5. This means that

$$
J_{1}=\left(\int_{-\delta}^{\delta} \frac{p\left(\lambda_{1}(r e(\alpha)), r e(\alpha)\right)}{-q_{x}\left(\lambda_{1}(r e(\alpha)), r e(\alpha)\right)} \lambda_{1}(r e(\alpha))^{-\nu-1} e(-k \alpha) d \alpha\right)\left(1+O_{G}\left(\eta_{G}^{-\nu}\right)\right) .
$$

We expand $\frac{p\left(\lambda_{1}(y), y\right)}{q_{x}\left(\lambda_{1}(y), y\right)}$ in a Taylor series around $y=r ; p(x, y)$ and $-q_{x}(x, y)$ are polynomials with positive coefficients, and we have $-q_{x}(1,0)=1$ and $p(1,0)=1$ (note that $\frac{p(x, 0)}{q(x, 0)}$ is the counting series for integers with sum of digits 0$)$. This means that $p(\lambda(y), y)$ and $-q_{x}(\lambda(y), y)$ can be bounded above and below for $y \leq 1$ (the bounds depending only on $G$ ), and their derivatives are also bounded. Therefore, we have

$$
\frac{p\left(\lambda_{1}(r e(\alpha)), r e(\alpha)\right)}{-q_{x}\left(\lambda_{1}(r e(\alpha)), r e(\alpha)\right)}=\frac{p(\lambda(r)), r)}{-q_{x}(\lambda(r), r)}\left(1+b(r) \alpha+O_{G}\left(\alpha^{2}\right)\right) \text {. }
$$

Likewise, we have

$$
\lambda_{1}(r e(\alpha))=\lambda(r)+2 \pi i \alpha r \lambda^{\prime}(r)-2 \pi^{2} r\left(\lambda^{\prime}(r)+r \lambda^{\prime \prime}(r)\right)+O_{G}\left(r \alpha^{3}\right) .
$$

Inserting yields

$$
\begin{aligned}
J_{1}= & \lambda(r)^{-\nu-1}\left(1+O_{G}\left(\eta_{G}^{-\nu}\right)\right) \int_{-\delta}^{\delta} \frac{p(\lambda(r)), r)}{-q_{x}(\lambda(r), r)}\left(1+b(r) \alpha+O_{G}\left(\alpha^{2}\right)\right) \\
& \exp \left(-\frac{2 \pi i \alpha \nu r \lambda^{\prime}(r)}{\lambda(r)}+\frac{2 \pi^{2} r \alpha^{2} \nu\left(\lambda(r) \lambda^{\prime}(r)+r \lambda(r) \lambda^{\prime \prime}(r)-r \lambda^{\prime}(r)^{2}\right)}{\lambda(r)^{2}}+O_{G}\left(r \alpha^{3} \nu\right)-2 \pi i k \alpha\right) d \alpha .
\end{aligned}
$$

$r$ was chosen in such a way that $\mu(r)=-\frac{r \lambda^{\prime}(r)}{\lambda(r)}=\frac{k}{\nu}$. Thus, the coefficients of $\alpha$ in the exponent cancel out. Furthermore, note that

$$
\frac{2 \pi^{2} r \nu\left(\lambda(r) \lambda^{\prime}(r)+r \lambda(r) \lambda^{\prime \prime}(r)-r \lambda^{\prime}(r)^{2}\right)}{\lambda(r)^{2}}=-2 \pi^{2} r \nu \mu^{\prime}(r) \leq-2 \pi^{2} r \nu c_{1}(G)<0
$$

by Lemma 3 . We write $D=2 \pi^{2} r \mu^{\prime}(r)$ and use the standard estimates

$$
\begin{gathered}
\int_{-\delta}^{\delta}\left(b(r) \alpha+O_{G}(\alpha)^{2}\right) \exp \left(-D \nu \alpha^{2}+O_{G}\left(r \alpha^{3} \nu\right)\right) d \alpha \\
=\int_{-\delta}^{\delta}\left(b(r) \alpha+O_{G}\left(\alpha^{2}\right)+O_{G}\left(r \alpha^{4} \nu\right)\right) \exp \left(-D \nu \alpha^{2}\right) d \alpha \\
=O_{G}\left(\int_{0}^{\delta} \alpha^{2} \exp \left(-D \nu \alpha^{2}\right) d \alpha\right)+O_{G}\left(r \nu \int_{0}^{\delta} \alpha^{4} \exp \left(-D \nu \alpha^{2}\right) d \alpha\right) \\
\int_{-\delta}^{\delta} \exp \left(-D \nu \alpha^{2}+O_{G}\left(r \nu \alpha^{3}\right)\right) d \alpha=\int_{-\delta}^{\delta} \exp \left(-D \nu \alpha^{2}\right) d \alpha+O_{G}\left(r \nu \int_{0}^{\delta} \alpha^{3} \exp \left(-D \nu \alpha^{2}\right)\right) \\
=\frac{1}{\sqrt{D \nu}}-2 \int_{\delta}^{\infty} \exp \left(-D \nu \alpha^{2}\right) d \alpha+O_{G}\left(r \nu \int_{0}^{\delta} \alpha^{3} \exp \left(-D \nu \alpha^{2}\right)\right) \\
\int_{0}^{\delta} \alpha^{p} \exp \left(-D \nu \alpha^{2}\right) d \alpha=(D \nu)^{-(p+1) / 2} \int_{0}^{\sqrt{D \nu} \delta} x^{p} \exp \left(-x^{2}\right) d x \\
\leq(D \nu)^{-(p+1) / 2} \int_{0}^{\infty} x^{p} \exp \left(-x^{2}\right) d x \\
=O\left((D \nu)^{-(p+1) / 2}\right)
\end{gathered}
$$


and

$$
\begin{aligned}
\int_{\delta}^{\infty} \exp \left(-D \nu \alpha^{2}\right) d \alpha & =\frac{1}{2 \sqrt{D \nu}} \int_{D \nu \delta^{2}}^{\infty} x^{-1 / 2} \exp (-x) d x \\
& \leq \frac{1}{2 D \nu \delta} \exp \left(-D \nu \delta^{2}\right) .
\end{aligned}
$$

Since $\mu^{\prime}(y)$ is bounded on $[0,1]$ by Lemma 3 , there are constants $c_{8}(G)$ and $c_{9}(G)$ such that

$$
c_{8}(G) \frac{k}{\nu} \leq r \leq c_{9}(G) \frac{k}{\nu} .
$$

Therefore, these estimates imply that

$$
J_{1}=\frac{p(\lambda(r), r)}{-\lambda(r) q_{x}(\lambda(r), r)}\left(2 \pi r \nu \mu^{\prime}(r)\right)^{-1 / 2} \lambda(r)^{-\nu}\left(1+O_{G}\left((D \nu)^{-1 / 2}\right)\right) .
$$

Finally, we estimate $J_{2}$ : by Lemma 7 ,

$$
\begin{aligned}
\left|J_{2}\right| & =\left|\int_{\delta \leq|\alpha| \leq 1 / 2}\left[x^{\nu}\right] \frac{p(x, r e(\alpha))}{q(x, r e(\alpha))} e(-k \alpha) d \alpha\right| \\
& \leq 2 c_{6}(G)\left[x^{\nu}\right] \frac{p(x, r)}{q(x, r)} \int_{\delta}^{1 / 2} \exp \left(-c_{7}(G) r \nu\|\alpha\|^{2}\right) d \alpha \\
& =O_{G}\left(\lambda(r)^{-\nu} \exp \left(-c_{7}(G) r \nu \delta^{2}\right)\right) .
\end{aligned}
$$

Altogether, we have established formula (14) for $\frac{k}{\nu} \leq \mu(1)$. We indicate how to extend it to the case $\frac{k}{\nu} \geq \mu(1)$ : if $A$ is taken as in Lemma 3 and $l=A \nu-k$, we have

$$
|F(k, \nu)|=\left[x^{\nu} y^{l}\right] \frac{p\left(x y^{A}, y^{-1}\right)}{q\left(x y^{A}, y^{-1}\right)} .
$$

The proof now goes along the same lines, with $\mu(y)$ replaced by $A-\mu\left(y^{-1}\right)$ and the roles of $y$ and $y^{-1}$ interchanged.

Corollary 9 There is a constant $c_{10}(G)$ depending only on $G$ such that the number of integers $\leq N$ with sum of digits $k$ is bounded below by

$$
c_{10}(G) \cdot \frac{p(\lambda(r), r)}{-\lambda(r) q_{x}(\lambda(r), r)} r^{-k} \lambda(r)^{-\nu} k^{-1 / 2}
$$

uniformly for $k \leq \mu(1) \nu, k \rightarrow \infty$, where $\nu+1$ is the number of digits of $N$.

Theorem 8 is a consequence of general theorems of Bender and Richmond [1,2] (see also Drmota [6]) in the case when $r$ is bounded above and below by positive constants. Equivalenty, $\frac{k}{\nu} \in[a, b]$ for constants $0<a<b<A$. It is easy to see that the sum of digits asymptotically follows a normal distribution with mean $\mu(1) \nu$ and variance $\mu^{\prime}(1) \nu$ : note first that $r^{-k} \lambda(r)^{-\nu}=\left(r^{\mu(r)} \lambda(r)\right)^{-\nu}$. The maximal value of $-\log \left(r^{\mu(r)} \lambda(r)\right)$ is achieved when the derivative is 0 , i.e.

$$
\frac{\mu(r)}{r}+\mu^{\prime}(r) \log (r)+\frac{\lambda^{\prime}(r)}{\lambda(r)}=\mu^{\prime}(r) \log (r)=0,
$$

which happens if $r=1$. The following corollary of Theorem 8 gives precise information:

Corollary 10 When $k$ is near the mean value, i.e. $\Delta=\mu(1) \nu-k=o(\nu)$, we have

$$
|F(k, \nu)|=\frac{p(\lambda(1), 1)}{-\lambda(1) q_{x}(\lambda(1), 1)} \lambda(1)^{-\nu} \cdot\left(2 \pi \nu \mu^{\prime}(1)\right)^{-1 / 2} \exp \left(-\frac{\Delta^{2}}{2 \nu \mu^{\prime}(1)}\right)\left(1+O_{G}\left(\frac{\Delta}{\nu}+\nu^{-1 / 2}\right)\right) .
$$


Proof. We set $\eta=1-r$ and use the Taylor expansion of $\mu$ around 1 to find that

$$
\eta=\frac{\Delta}{\nu \mu^{\prime}(1)}+O_{G}\left(\frac{\Delta^{2}}{\nu^{2}}\right)
$$

Then,

$$
r^{-k}=\exp (-k \log (1-\eta))=\exp \left(k \eta+\frac{1}{2} k \eta^{2}+O\left(k \eta^{3}\right)\right)
$$

and

$$
\lambda(r)^{-\nu}=\lambda(1)^{-\nu} \exp \left(\nu\left(\frac{\lambda^{\prime}(1)}{\lambda(1)} \eta+\frac{\lambda^{\prime}(1)^{2}-\lambda(1) \lambda^{\prime \prime}(1)}{2 \lambda(1)^{2}} \eta^{2}+O_{G}\left(\eta^{3}\right)\right)\right) .
$$

Furthermore,

$$
\frac{p(\lambda(r), r)}{-\lambda(r) q_{x}(\lambda(r), r)}\left(2 \pi r \nu \mu^{\prime}(r)\right)^{-1 / 2}=\frac{p(\lambda(1), 1)}{-\lambda(1) q_{x}(\lambda(1), 1)}\left(2 \pi \nu \mu^{\prime}(1)\right)^{-1 / 2}\left(1+O_{G}\left(\frac{\Delta}{\nu}\right)\right) .
$$

We insert $k=\mu(1) \nu-\Delta$ and use the formula

$$
\mu^{\prime}(y)=\frac{y \lambda^{\prime}(y)^{2}-y \lambda(y) \lambda^{\prime \prime}(y)-\lambda(y) \lambda^{\prime}(y)}{\lambda(y)^{2}}
$$

to obtain the stated result.

REMARK. Note that $\frac{p(\lambda(1), 1)}{-\lambda(1) q_{x}(\lambda(1), 1)} \lambda(1)^{-\nu}$ is (asymptotically) the number of all integers with an expansion of $\leq \nu$ digits.

Corollary 11 If $k$ is small, i.e. $k=o(\nu)$, we have

$$
|F(k, \nu)|=(2 \pi k)^{-1 / 2} \exp \left(-k \log \frac{k}{\nu}+k+\frac{1-\lambda^{\prime \prime}(0)}{2} \cdot \frac{k^{2}}{\nu}+O_{G}\left(\frac{k^{3}}{\nu^{2}}+\frac{1}{\sqrt{k}}\right)\right) .
$$

REMARK. It is easy to check that

$$
\lambda^{\prime \prime}(0)= \begin{cases}4 & d=2, a_{1}=a_{2}=1, \\ 2 & d=1, a_{1}=2 \text { or } d>2, a_{1}=a_{2}=\ldots=a_{d}=1, \\ 0 & \text { otherwise }\end{cases}
$$

Proof. We see that

$$
r=\frac{k}{\nu}-\frac{\mu^{\prime \prime}(0)}{2}\left(\frac{k}{\nu}\right)^{2}+O_{G}\left(\left(\frac{k}{\nu}\right)^{3}\right),
$$

since $\mu^{\prime}(0)=1$. This gives us

$$
r \mu^{\prime}(r)=\frac{k}{\nu}+\frac{\mu^{\prime \prime}(0)}{2}\left(\frac{k}{\nu}\right)^{2}+O_{G}\left(\left(\frac{k}{\nu}\right)^{3}\right)
$$

and

$$
\lambda(r)=1-r+\frac{\lambda^{\prime \prime}(0)}{2} r^{2}+O_{G}\left(r^{3}\right)=1-\frac{k}{\nu}+\frac{\lambda^{\prime \prime}(0)+\mu^{\prime \prime}(0)}{2}\left(\frac{k}{\nu}\right)^{2}+O_{G}\left(\left(\frac{k}{\nu}\right)^{3}\right) .
$$

Therefore,

$$
\begin{gathered}
\frac{p(\lambda(r), r)}{-\lambda(r) q_{x}(\lambda(r), r)}=\frac{p(\lambda(0), 0)}{-\lambda(0) q_{x}(\lambda(0), 0)}\left(1+O_{G}\left(\frac{k}{\nu}\right)\right)=1+O_{G}\left(\frac{k}{\nu}\right), \\
2 \pi r \mu^{\prime}(r) \nu=2 \pi k\left(1+O_{G}\left(\frac{k}{\nu}\right)\right), \\
-k \log r=-k \log \frac{k}{\nu}+\frac{\mu^{\prime \prime}(0)}{2} \cdot \frac{k^{2}}{\nu}+O_{G}\left(\frac{k^{3}}{\nu^{2}}\right),
\end{gathered}
$$


and

$$
-\nu \log \lambda(r)=k-\frac{\lambda^{\prime \prime}(0)+\mu^{\prime \prime}(0)-1}{2} \cdot \frac{k^{2}}{\nu}+O_{G}\left(\frac{k^{3}}{\nu^{2}}\right) .
$$

Inserting in (14) yields the stated result.

EXAMPLE. It is not difficult to check that our result agrees with (3) in the case $d=1, a_{1}=g$. We will consider the classical Zeckendorf expansion $\left(d=2, a_{1}=a_{2}=1, G_{0}=1, G_{1}=2\right)$ as another example. In this case, we have

$$
p(x, y)=1+x y, q(x, y)=1-x-y x^{2},
$$

yielding

$$
\lambda(y)=\frac{1}{2 y}(\sqrt{1+4 y}-1), \mu(y)=\frac{1}{2}\left(1-\frac{1}{\sqrt{1+4 y}}\right) .
$$

If we set $\frac{k}{n}=\gamma$, we obtain

$$
|F(k, \nu)| \sim \sqrt{\frac{(1-\gamma)^{3}}{2 \pi \gamma(1-2 \gamma)^{3} \nu}} \cdot\left(\frac{(1-\gamma)^{1-\gamma}}{\gamma^{\gamma}(1-2 \gamma)^{1-2 \gamma}}\right)^{\nu} .
$$

The mean value is given by $\mu \nu=\mu(1) \nu=\frac{1}{2}\left(1-\frac{1}{\sqrt{5}}\right) \nu$, the variance by $\sigma^{2} \nu=\mu^{\prime}(1) \nu=5^{-3 / 2} \nu$.

\section{Distribution in residue classes}

The aim of this section is to prove that $F(k, \nu)$ is well-distributed in residue classes modulo $m$ provided that $m$ is not too large and there is no prime divisor $P$ of $m$ such that $G_{n}$ is constant modulo $P$ for all but finitely many values of $n$.

Theorem 12 Let $V(k, N)$ be the set of integers $\leq N$ with $G$-ary sum of digits $k$. There exist positive constants $k_{0}(G), c_{11}(G), c_{12}(G), c_{13}(G)$ (depending on $G$ only) such that for all $l=\max (k, A \nu-k) \geq$ $k_{0}(G)$ ( $\nu$ denoting the number of $G$-ary digits of $N$ ), $2 \leq m<\exp \left(c_{11}(G) l^{1 / 2}\right), h \in \mathbb{Z}$, for which there is no prime divisor $P$ of $m$ such that $\left(G_{n}\right)$ is constant modulo $P$ for all but finitely many values of $n$, we have

$$
||\{n \in V(k, N): n \equiv h \quad \bmod m\}\left|-\frac{1}{m}\right| V(k, N)||<\frac{c_{11}(G)}{m}|V(k, N)| \exp \left(-c_{12}(G) \frac{k}{\log m}\right) .
$$

Remark. The condition on the prime factors of $m$ is a necessary one. If $\left(G_{n}\right)$ was constant modulo $P$ for all but finitely many values of $n$, the restriction on the sum of digits would imply a condition on the residues modulo $P$. Note that $\left(g^{n}\right)_{n \geq 0}$ is constant modulo $P$ for all but finitely many values of $n$ if and only if $P \mid g(g-1)$.

Proof. We follow the lines of [19] again. Again, we consider the case $k \leq \mu(1) \nu$ only. if

$$
D(z, \gamma)=\sum_{n=1}^{N} z^{s_{G}(n)} e(n \gamma),
$$

where $z \in \mathbb{C}, \gamma \in \mathbb{R}$, we have

$$
\frac{1}{m} \sum_{p=1}^{m} e\left(-\frac{h p}{m}\right) D\left(z, \frac{p}{m}\right)=\sum_{\substack{1 \leq n \leq N \\ n \equiv h \text { mod } m}} z^{s_{G}(n)} .
$$


Now we take $r$ as in the proof of Theorem 8 and obtain

$$
\begin{aligned}
\left|\left\{n \leq N: s_{G}(n)=k, n \equiv h \bmod m\right\}\right| & =r^{-k} \int_{0}^{1} e(-k \beta) \sum_{\substack{1 \leq n \leq N \\
n \equiv h}}(r e(\beta))^{s_{G}(n)} d \beta \\
& =\frac{1}{m} r^{-k} \sum_{p=1}^{m} \int_{0}^{1} e\left(-k \beta-\frac{h p}{m}\right) D\left(\operatorname{re}(\beta), \frac{p}{m}\right) d \beta .
\end{aligned}
$$

Obviously, the summand corresponding to $p=m$ equals $\frac{1}{m}|V(k, N)|$. Thus we have to estimate

$$
\frac{1}{m} r^{-k} \sum_{p=1}^{m-1} \int_{0}^{1}\left|D\left(r e(\beta), \frac{p}{m}\right)\right| d \beta
$$

We write $N$ in base- $G$ representation:

$$
N=\sum_{j=0}^{L(N)} \epsilon_{j} G_{j}=\sum_{i=1}^{t} \epsilon_{\nu_{i}} G_{\nu_{i}}
$$

where $\nu_{1}>\nu_{2}>\ldots>\nu_{t}$ and all $\epsilon_{\nu_{i}}$ are positive (i.e., we neglect all digits 0 in the base- $G$ representation). Then, the set $\{0, \ldots, N\}$ can be partitioned into sets $A_{l}$, where $A_{l}$ is the set of integers representable as

$$
\sum_{i=1}^{l-1} \epsilon_{\nu_{i}} G_{\nu_{i}}+a G_{\nu_{l}}+b
$$

where $0 \leq a \leq \epsilon_{\nu_{l}}-1$ and $b$ is an arbitrary integer with $\leq \nu_{l} G$-ary digits. Let the set of all such integers be denoted by $B_{\nu_{l}}$. Additionally, we set $A_{t+1}=\{N\}$. Then we have

$$
\begin{aligned}
1+D(r e(\beta), \gamma)= & \sum_{n=0}^{N}(r e(\beta))^{s_{G}(n)} e(n \gamma) \\
= & \sum_{l=1}^{t+1} \sum_{n \in A_{l}}(r e(\beta))^{s_{G}(n)} e(n \gamma) \\
= & (r e(\beta))^{s_{G}(N)} e(N \gamma)+\sum_{l=1}^{t} \sum_{a=0}^{\epsilon_{\nu_{l}}-1} \sum_{b \in B_{\nu_{l}}}(r e(\beta))^{\epsilon_{\nu_{1}}+\ldots+\epsilon_{\nu_{l-1}}+a+s_{G}(b)} \\
& e\left(\left(\sum_{i=1}^{l-1} \epsilon_{\nu_{i}} G_{\nu_{i}}+a G_{\nu_{l}}+b\right) \gamma\right),
\end{aligned}
$$

from which it follows that

$$
\begin{aligned}
|D(r e(\beta), \gamma)| & \leq 2+\sum_{l=1}^{t} r^{\epsilon_{\nu_{1}}+\ldots+\epsilon_{\nu_{l-1}}}\left|\sum_{a=0}^{\epsilon_{\nu_{l}}-1}\left(r e\left(\beta+G_{\nu_{l}} \gamma\right)\right)^{a}\right|\left|\sum_{b \in B_{\nu_{l}}}(r e(\beta))^{s_{G}(b)} e(b \gamma)\right| \\
& \leq 2+\sum_{l=1}^{t} r^{l-1} \epsilon_{\nu_{l}}\left|\sum_{b \in B_{\nu_{l}}}(r e(\beta))^{s_{G}(b)} e(b \gamma)\right|
\end{aligned}
$$

We write

$$
u_{\nu}(\beta, \gamma):=\sum_{b \in B_{\nu}}(r e(\beta))^{s_{G}(b)} e(b \gamma) .
$$

Then we see that $u_{\nu}$ satisfies a recursive relation:

Lemma 13 For $\nu \geq 2 d$, we have

$$
u_{\nu}(\beta, \gamma)=\sum_{i=1}^{d}\left(\sum_{j=0}^{a_{i}-1}\left(r e\left(\beta+G_{\nu-i} \gamma\right)\right)^{j}\right)\left(\prod_{l=1}^{i-1}\left(r e\left(\beta+G_{\nu-l} \gamma\right)\right)^{a_{l}}\right) u_{\nu-i}(\beta, \gamma) .
$$


Proof. This is proved in the same way as Proposition 2: note that appending a sequence of the form $\left(\epsilon, a_{i-1}, \ldots, a_{1}\right)$ with $\epsilon<a_{i}$ to a good sequence of length $\nu-i$ gives a factor of

$$
(r e(\beta))^{a_{1}+\ldots+a_{i-1}+\epsilon} e\left(\left(G_{\nu-1} a_{1}+\ldots+G_{\nu-i+1} a_{i-1}+G_{\nu-i} \epsilon\right) \gamma\right) .
$$

The recurrence can be used to prove an analogue of Lemma 7:

Lemma 14 There exist constants $c_{14}(G), c_{15}(G)$ depending only on $G$ such that

$$
u_{\nu}(\beta, \gamma) \leq c_{14}(G) \exp \left(-c_{15}(G) r \sum_{n=0}^{\nu-1}\left\|\beta+G_{n} \gamma\right\|^{2}\right) u_{\nu}(0,0)
$$

for all $0<r \leq 1$ and all $\beta, \gamma \in \mathbb{R}$.

Proof. This is done almost analogously to the proof of Lemma 7. For $a_{1}>1$ (the other case is similar), we have

$$
\left|u_{\nu}(\beta, \gamma)\right| \leq\left(1-\frac{2 r}{a_{1}}\left\|\beta+G_{\nu-1} \gamma\right\|^{2}\right) \sum_{j=0}^{a_{1}-1} r^{j}\left|u_{\nu-1}(\beta, \gamma)\right|+\sum_{i=2}^{d} \sum_{j=0}^{a_{i}-1} r^{j} \prod_{l=1}^{i-1} r^{a_{l}}\left|u_{\nu-i}(\beta, \gamma)\right|
$$

by the same argument as in Lemma 7. If we define $U_{\nu}(\beta, \gamma)$ by $U_{\nu}(\beta, \gamma)=u_{\nu}(0,0)$ for $\nu<d$ and

$$
U_{\nu}(\beta, \gamma)=\left(1-\frac{2 r}{a_{1}}\left\|\beta+G_{\nu-1} \gamma\right\|^{2}\right) \sum_{j=0}^{a_{1}-1} r^{j} U_{\nu-1}(\beta, \gamma)+\sum_{i=2}^{d} \sum_{j=0}^{a_{i}-1} r^{j} \prod_{l=1}^{i-1} r^{a_{l}} U_{\nu-i}(\beta, \gamma)
$$

we know that $\left|u_{\nu}(\beta, \gamma)\right| \leq U_{\nu}(\beta, \gamma)$ for all $\nu$, and the argument of Lemma 7 shows that

$$
U_{\nu}(\beta, \gamma) \leq\left(1-\frac{2 r}{a_{1} d}\left\|\beta+G_{\nu-1} \gamma\right\|^{2}\right) \sum_{i=1}^{d} \sum_{j=0}^{a_{i}-1} r^{j} \prod_{l=1}^{i-1} r^{a_{l}} U_{\nu-i}(\beta, \gamma)
$$

Write $C_{i}:=\sum_{j=0}^{a_{i}-1} r^{j} \prod_{l=1}^{i-1} r^{a_{l}}$. For a sequence $\mathbf{x}=\left(x_{n}\right)_{n \geq 0}$ with $1 \geq x_{n} \geq 1-\frac{r}{2}$, define $W_{\nu}(\mathbf{x})$ by $W_{\nu}(\mathbf{x})=u_{\nu}(0,0)$ for $\nu<d$ and

$$
W_{\nu}(\mathbf{x})=x_{\nu} \sum_{i=1}^{d} C_{i} W_{\nu-i}(\mathbf{x})
$$

Since $x_{\nu} C_{1} \geq\left(1-\frac{r}{2}\right)(1+r)=1+\frac{r(1-r)}{2} \geq 1$, we know that $W_{\nu}(\mathbf{x})$ is increasing, and we also know that the $C_{i}$ are decreasing, so $C_{i} W_{\nu-i}(\mathbf{x})$ is always decreasing. Let $\mathbf{x}^{(n)}$ be the sequence $\mathbf{x}$ with 1 at the place of $x_{n}$. We claim that

$$
W_{\nu}(\mathbf{x}) \leq\left(1-\frac{1-x_{n}}{d}\right) W_{\nu}\left(\mathbf{x}^{(n)}\right)
$$

holds for $\nu \geq n$. This is trivial for $\nu=n$, since we have

$$
W_{n}(\mathbf{x})=x_{n} W_{n}\left(\mathbf{x}^{(n)}\right)
$$


and $\left(1-\frac{1-x_{n}}{d}\right) \geq x_{n}$. We proceed by induction: for $1 \leq j \leq d-1$, we have

$$
\begin{aligned}
W_{n+j}(\mathbf{x}) & =\sum_{i=1}^{j-1} C_{i} W_{n+j-i}(\mathbf{x})+C_{j} W_{n}(\mathbf{x})+\sum_{i=j+1}^{d} C_{i} W_{n+j-i}(\mathbf{x}) \\
& \leq\left(1-\frac{1-x_{n}}{d}\right) \sum_{i=1}^{j-1} C_{i} W_{n+j-i}\left(\mathbf{x}^{(n)}\right)+x_{n} C_{j} W_{n}\left(\mathbf{x}^{(n)}\right)+\sum_{i=j+1}^{d} C_{i} W_{n+j-i}\left(\mathbf{x}^{(n)}\right) \\
& \leq\left(1-\frac{1-x_{n}}{d}\right) \sum_{i=1}^{j-1} C_{i} W_{n+j-i}\left(\mathbf{x}^{(n)}\right)+\frac{d-j+x_{n}}{d-j+1} \sum_{i=j}^{d} C_{i} W_{n+j-i}\left(\mathbf{x}^{(n)}\right) \\
& \leq\left(1-\frac{1-x_{n}}{d}\right) \sum_{i=1}^{d} C_{i} W_{n+j-i}\left(\mathbf{x}^{(n)}\right) \\
& =\left(1-\frac{1-x_{n}}{d}\right) W_{n+j}\left(\mathbf{x}^{(n)}\right) .
\end{aligned}
$$

For $j \geq d$, the induction is even simpler. Another straightforward induction shows that

$$
W_{\nu}(\mathbf{x}) \leq \prod_{j=d}^{\nu}\left(1-\frac{1-x_{j}}{d}\right) W_{\nu}(\mathbf{1}),
$$

where 1 is the sequence consisting only of 1's. In our special case, we take $x_{n}=1-\frac{2 r}{a_{1} d}\left\|\beta+G_{n-1} \gamma\right\|^{2}$ to show that

$$
\begin{aligned}
U_{\nu}(\beta, \gamma) & \leq \prod_{n=d}^{\nu}\left(1-\frac{2 r}{a_{1} d^{2}}\left\|\beta+G_{n-1} \gamma\right\|^{2}\right) u_{\nu}(0,0) \\
& \leq\left(1-\frac{1}{2 a_{1} d^{2}}\right)^{1-d} \prod_{n=1}^{\nu}\left(1-\frac{2 r}{a_{1} d^{2}}\left\|\beta+G_{n-1} \gamma\right\|^{2}\right) u_{\nu}(0,0) \\
& \leq\left(1-\frac{1}{2 a_{1} d^{2}}\right)^{1-d} \exp \left(-\frac{2 r}{a_{1} d^{2}} \sum_{n=0}^{\nu-1}\left\|\beta+G_{n} \gamma\right\|^{2}\right) u_{\nu}(0,0),
\end{aligned}
$$

which finally proves the claim.

Lemma 15 Let $m, \rho \in \mathbb{N}$ and $1 \leq p \leq m-1$. If there is no prime divisor $P$ of $m$ such that the sequence $G_{n}$ is constant modulo $P$ for all but finitely many values of $n$, we have

$$
\sum_{n=0}^{\rho-1}\left\|\beta+G_{n} \frac{j}{m}\right\|^{2} \geq c_{16}(G) \frac{\rho}{\log m}+O_{G}(1) .
$$

Proof. Without loss of generality, we may assume that $(p, m)=1$ (cancellation of common factors only improves the bound, and the conditions keep true). First, we show that there exist constants $c_{17}(G)$ and $c_{18}(G)$ such that, among any set of $c_{17}(G)+c_{18}(G) \log m$ consequent integers, there is an integer $n$ such that

$$
\left\|\left(G_{n+1}-G_{n}\right) \frac{p}{m}\right\| \geq \frac{1}{2\left(a_{1}+\ldots+a_{d}\right)} .
$$

For this purpose, we define a sequence $\left(A_{n}\right)_{n \geq 0}$ by $A_{n} \equiv\left(G_{n+1}-G_{n}\right) p \bmod m$ and $-\frac{m}{2}<A_{n} \leq \frac{m}{2}$. We want to show that there are constants $c_{17}(G)$ and $c_{18}(G)$ such that for all $I \geq 0$, there is an $n<$ $c_{17}(G)+c_{18}(G) \log m$ with

$$
\left\|\frac{A_{I+n}}{m}\right\| \geq \frac{1}{2\left(a_{1}+\ldots+a_{d}\right)}
$$


First, of all, we will take $c_{17}(G) \geq d$. Consider the values $A_{I}, A_{I+1}, \ldots, A_{I+d-1}$. If one of them has absolute value $\geq \frac{m}{2\left(a_{1}+\ldots+a_{d}\right)}$, we are done. Otherwise, define the sequence $\left(B_{n}\right)_{n \geq 0}$ by $B_{n}=A_{I+n}$ $(n=0, \ldots, d-1)$ and

$$
B_{n+d}=a_{1} B_{n+d-1}+a_{2} B_{n+d-2}+\ldots+a_{d} B_{n} .
$$

Note that $B_{n} \equiv A_{I+n}$ for all values of $n$. Now we use a result of Brauer [4] that was also applied in [21]: The characteristic polynomial

$$
x^{d}-a_{1} x^{d-1}-\ldots-a_{d}
$$

has a dominating root $\theta \in\left[a_{1}, a_{1}+1\right.$ ) that is a Pisot number, i.e., all conjugates $\theta_{2}, \ldots, \theta_{d}$ (if $d>1$ ) have modulus $<1$. Thus, we can express $B_{n}$ by an explicit formula:

$$
B_{n}=\beta \theta^{n}+\sum_{i=2}^{d} \beta_{i} n^{\delta(i)} \theta_{i}^{n},
$$

where the $\beta_{i}$ are linear combinations of the initial values $B_{0}, B_{1}, \ldots, B_{d-1}$ (with algebraic coefficients depending only on the characteristic polynomial). Therefore, there exist constants $c_{19}(G)$ and $\kappa_{3}(G)$ such that

$$
\left|B_{n}-\beta \theta^{n}\right| \leq c_{19}(G) m n^{\kappa_{3}(G)}\left|\theta_{2}\right|^{n} .
$$

The coefficient $\beta$ is also a linear combination of the initial values, i.e. it is of the form

$$
x_{0} B_{0}+\ldots+x_{d-1} B_{d-1},
$$

where the $x_{i}$ are algebraic numbers depending on the characteristic polynomial. By a result of Schmidt (cf. [7, Theorem 2.1]), the inequality

$$
0<\left|x_{0} B_{0}+\ldots+x_{d-1} B_{d-1}\right|<M^{-d+1-\epsilon}
$$

with $\left|B_{n}\right| \leq M$ has only finitely many solutions for every $\epsilon>0$; therefore, there are constants $c_{20}(G)>0$ and $\kappa_{4}(G)$ such that either

$$
\beta=x_{0} B_{0}+\ldots+x_{d-1} B_{d-1}=0
$$

or

$$
|\beta|=\left|x_{0} B_{0}+\ldots+x_{d-1} B_{d-1}\right| \geq c_{20}(G) M^{-\kappa_{4}(G)}
$$

whenever $\left|B_{0}\right|, \ldots,\left|B_{d-1}\right| \leq M$. We know that $\beta$ cannot be 0 , since then we would have $\lim _{n \rightarrow \infty} B_{n}=0$, i.e. $A_{n} \equiv 0 \bmod m$ for all but finitely many values of $n$. This contradicts the assumptions on $G$ : as $(p, m)=1, G_{n}$ would be constant modulo $m$ for all but finitely many values of $n$. Therefore, since $\left|B_{n}\right| \leq \frac{m}{2\left(a_{1}+\ldots+a_{d}\right)}$ for $0 \leq n \leq d-1,|\beta| \geq c_{21}(G) m^{-\kappa_{4}}$, where $c_{21}>0$ depends only on $G$. It follows that

$$
\left|B_{n}\right| \geq c_{21}(G) m^{-\kappa_{4}} \theta^{n}-c_{19}(G) m n^{\kappa_{3}(G)}\left|\theta_{2}\right|^{n}
$$

for all $n$; there are constants $c_{22}(G)$ and $c_{23}(G)$ such that

$$
c_{21}(G) m^{-\kappa_{4}(G)} \theta^{n}-c_{19}(G) m n^{\kappa_{3}(G)}\left|\theta_{2}\right|^{n}
$$

for all $n \geq c_{22}(G) \log m+c_{23}(G)$. Thus, $B_{n} \geq \frac{m}{2\left(a_{1}+\ldots+a_{d}\right)}$ for some $n \leq c_{22}(G) \log m+c_{23}(G)$; for the smallest index $n$ for which this is true, we must also have $B_{n} \leq \frac{m}{2}$, so

$$
\left\|\frac{A_{I+n}}{m}\right\|=\left\|\frac{B_{n}}{m}\right\| \geq \frac{1}{2\left(a_{1}+\ldots+a_{d}\right)} .
$$


This proves the claim, and the lemma is a simple consequence if we make use of the inequality

$$
\left\|\beta+G_{k+1} \frac{p}{m}\right\|^{2}+\left\|\beta+G_{k} \frac{p}{m}\right\|^{2} \geq \frac{1}{2}\left\|G_{k+1} \frac{p}{m}-G_{k} \frac{p}{m}\right\|^{2} .
$$

We turn back to the proof of Theorem 12. By the preceding lemmas, there are constants $c_{24}(G)$ and $c_{25}(G)$ such that

$$
u_{\nu}\left(\beta, \frac{p}{m}\right) \leq c_{24}(G) \exp \left(-c_{25}(G) \frac{r \nu}{\log m}\right) u_{\nu}(0,0) .
$$

Therefore, since $u_{\nu_{l}}(0,0)=\sum_{b \in B_{\nu_{l}}} r^{s_{G}(b)}$, we have

$$
\left|D\left(r e(\beta), \frac{p}{m}\right)\right| \leq c_{24}(G)\left(\sum_{l=1}^{t} r^{l-1} \epsilon_{\nu_{l}} \exp \left(-c_{25}(G) \frac{r \nu_{l}}{\log m}\right) \sum_{b \in B_{\nu_{l}}} r^{s_{G}(b)}\right)+O_{G}(1) .
$$

We divide the sum on the right side into two parts by defining the integer $q$ for which $\nu_{q} \geq \nu / 2>\nu_{q+1}$ $\left(\right.$ set $\left.\nu_{t+1}=0\right)$ : the first part is defined by

$$
S_{1}:=\sum_{l=1}^{q} r^{l-1} \epsilon_{\nu_{l}} \exp \left(-c_{25}(G) \frac{r \nu_{l}}{\log m}\right) \sum_{b \in B_{\nu_{l}}} r^{s_{G}(b)} \leq c_{26}(G) \exp \left(-c_{25}(G) \frac{r \nu / 2}{\log m}\right) \sum_{l=1}^{q} r^{l-1} \sum_{b \in B_{\nu_{l}}} r^{s_{G}(b)}
$$

where $c_{26}(G)$ is the largest possible digit that can appear in a $G$-ary expansion. Next, we observe that

$$
\sum_{b \in B_{\nu_{l}}} r^{s_{G}(b)}=\left[x^{\nu_{l}}\right] \frac{p(x, r)}{q(x, r)} .
$$

By Corollary 5, this equals

$$
\sum_{b \in B_{\nu_{l}}} r^{s_{G}(b)}=-\frac{p(\lambda(r), r)}{q_{x}(\lambda(r), r)} \lambda(r)^{-\nu_{l}-1}\left(1+O_{G}\left(\eta_{G}^{-\nu_{l}}\right)\right)
$$

so that we obtain

$$
\begin{aligned}
S_{1} & \leq c_{26}(G) \exp \left(-c_{25}(G) \frac{r \nu / 2}{\log m}\right) \sum_{l=1}^{q} r^{l-1} \cdot\left(-\frac{p(\lambda(r), r)}{q_{x}(\lambda(r), r)}\right) \lambda(r)^{-\nu_{l}-1}\left(1+O_{G}\left(\eta_{G}^{-\nu_{l}}\right)\right) \\
& =c_{26}(G) \exp \left(-c_{25}(G) \frac{r \nu / 2}{\log m}\right)\left(1+O_{G}\left(\eta_{G}^{-\nu / 2}\right)\right) \cdot\left(-\frac{p(\lambda(r), r)}{\lambda(r) q_{x}(\lambda(r), r)}\right) \lambda(r)^{-\nu} \cdot \sum_{l=1}^{q} r^{l-1} \lambda(r)^{\nu-\nu_{l}} \\
& \leq c_{26}(G) \exp \left(-c_{25}(G) \frac{r \nu / 2}{\log m}\right)\left(1+O_{G}\left(\eta_{G}^{-\nu / 2}\right)\right) \cdot\left(-\frac{p(\lambda(r), r)}{\lambda(r) q_{x}(\lambda(r), r)}\right) \lambda(r)^{-\nu} \cdot \sum_{j=0}^{\infty}(r \lambda(r))^{j}
\end{aligned}
$$

If $a_{1} \geq 2$, we have $q\left(\left(1+r+\ldots+r^{a_{1}-1}\right)^{-1}, r\right)<0$ and thus $\lambda(r) \leq\left(1+r+\ldots+r^{a_{1}-1}\right)^{-1} \leq(1+r)^{-1}$, which in turn means that $r \lambda(r) \leq \frac{r}{1+r} \leq \frac{1}{2}$. If $a_{1}=1$, we also have $a_{2}=1$ and thus $q\left(\frac{\sqrt{1+4 r}-1}{2 r}, r\right) \leq 0$, so we obtain $r \lambda(r) \leq \frac{\sqrt{1+4 r}-1}{2} \leq \frac{\sqrt{5}-1}{2}$. This means that the infinite sum converges and is bounded by $\frac{3+\sqrt{5}}{2}$. Together with Corollary 9 , we obtain

$$
S_{1}=O_{G}\left(|V(k, N)| r^{k} k^{1 / 2} \exp \left(-c_{25}(G) \frac{r \nu / 2}{\log m}\right)\right) .
$$

The other part of the sum,

$$
S_{2}:=\sum_{l=q+1}^{t} r^{l-1} \epsilon_{\nu_{l}} \exp \left(-c_{25}(G) \frac{r \nu_{l}}{\log m}\right) \sum_{b \in B_{\nu_{l}}} r^{s_{G}(b)}
$$


can be estimated as follows:

$$
\begin{aligned}
S_{2} & \leq c_{26}(G) \sum_{l=q+1}^{t} r^{l-1} \sum_{b \in B_{\nu_{l}}} r^{s_{G}(b)} \\
& =c_{26}(G) \sum_{l=q+1}^{t} r^{l-1} \cdot\left(-\frac{p(\lambda(r), r)}{q_{x}(\lambda(r), r)}\right) \lambda(r)^{-\nu_{l}-1}\left(1+O_{G}\left(\eta_{G}^{-\nu_{l}}\right)\right) \\
& \leq c_{26}(G) \cdot\left(-\frac{p(\lambda(r), r)}{\lambda(r) q_{x}(\lambda(r), r)}\right) \sum_{i=1}^{t-q} r^{i-1} \lambda(r)^{-\nu / 2+(i-1)}\left(1+O_{G}(1)\right) \\
& \leq c_{26}(G) \cdot\left(-\frac{p(\lambda(r), r)}{\lambda(r) q_{x}(\lambda(r), r)}\right) \lambda(r)^{-\nu / 2} \sum_{j=0}^{\infty}(r \lambda(r))^{j}\left(1+O_{G}(1)\right)
\end{aligned}
$$

and thus

$$
S_{2}=O_{G}\left(|V(k, N)| r^{k} k^{1 / 2} \lambda(r)^{\nu / 2}\right) .
$$

It is known that $\mu^{\prime}(y)$ is bounded above and below by positive constants depending only on $G$, which means that there are constants $c_{8}, c_{9}$ such that

$$
c_{8}(G) \frac{k}{\nu} \leq r \leq c_{9}(G) \frac{k}{\nu}
$$

Furthermore, $\lambda^{\prime}(y)=-\frac{\lambda(y) \mu(y)}{y}$ is strictly negative on $(0,1]$ with $\lim _{y \rightarrow 0+} \lambda^{\prime}(y)=-1$, so it is bounded above and below by negative constants. So there are constants $c_{27}(G)$ and $c_{28}(G)$ such that

$$
c_{27}(G) \frac{k}{\nu} \leq \lambda(0)-\lambda(r)=1-\lambda(r) \leq c_{28}(G) \frac{k}{\nu}
$$

and thus

$$
\lambda(r)^{\nu / 2} \leq\left(1-c_{27}(G) \frac{k}{\nu}\right)^{\nu / 2} \leq \exp \left(-\frac{c_{27}(G)}{2} k\right)
$$

Altogether, we obtain

$$
S_{1}+S_{2}=O_{G}\left(|V(k, N)| r^{k} k^{1 / 2}\left(\exp \left(-c_{29}(G) \frac{k}{\log m}\right)+\exp \left(-\frac{c_{27}(G)}{2} k\right)\right)\right),
$$

which proves Theorem 12 .

REMARK. As an example, we note that, since the Fibonacci numbers clearly satisfy the condition for any modulus, the set of integers with a fixed number of 1's in the Zeckendorf representation is well-distributed modulo any integer modulus. As in [19], Theorem 12 can also be used to prove the following:

Corollary 16 If $z \in \mathbb{N}, z \geq 2$, then there are constants $N_{0}(G), c_{30}(G), c_{31}(G)$ (depending on $G$ and $z$ ) such that for all $N \geq N_{0}(G)$ and all $k$ with

$$
|\mu(1) \nu-k|<c_{30}(G)(\log N)^{3 / 4},
$$

where $\nu+1$ is the number of $G$-ary digits of $N$, the number of integers in $V(k, N)$ which are not divisible by the $z$-th power of a prime $P$ in the set

$$
\mathcal{P}:=\{P: P \text { prime, } P \text { satisfies the condition of Theorem } 12\}
$$

is given by

$$
\left(\zeta(z) \prod_{P \in \mathcal{P}}\left(1-\frac{1}{p^{z}}\right)\right)^{-1}|V(k, N)|\left(1+O\left(\exp \left(-c_{31}(G)(\log N)^{1 / 2}\right)\right)\right) .
$$




\section{Acknowledgment}

This work was supported by Austrian Science Fund project no. S-8307-MAT.

\section{References}

[1] E. A. Bender, Central and local limit theorems applied to asymptotic enumeration, J. Combinatorial Theory Ser. A 15 (1973), 91-111.

[2] E. A. Bender and L. B. Richmond, Central and local limit theorems applied to asymptotic enumeration. II. Multivariate generating functions, J. Combin. Theory Ser. A 34(3) (1983), 255-265.

[3] D. Berend and C. Frougny, Computability by finite automata and Pisot bases, Math. Systems Theory 27(3) (1994), 275-282.

[4] A. Brauer, On algebraic equations with all but one root in the interior of the unit circle, Math. Nachr. 4 (1951), 250-257.

[5] H. Delange, Sur la fonction sommatoire de la fonction "somme des chiffres", Enseignement Math. (2) 21(1) (1975), 31-47.

[6] M. Drmota, Asymptotic distributions and a multivariate Darboux method in enumeration problems, J. Combin. Theory Ser. A 67(2) (1994), 169-184.

[7] B. Edixhoven and J.-H. Evertse, editors, Diophantine approximation and abelian varieties, volume 1566 of Lecture Notes in Mathematics (Springer, Berlin, 1993). Introductory lectures, Papers from the conference held in Soesterberg, April 12-16, 1992.

[8] P. Erdős, C. Mauduit, and A. Sárközy, On arithmetic properties of integers with missing digits. I. Distribution in residue classes, J. Number Theory 70(2) (1998), 99-120.

[9] P. Erdős, C. Mauduit, and A. Sárközy, On arithmetic properties of integers with missing digits. II. Prime factors, Discrete Math. 200(1-3) (1999), 149-164. Paul Erdős memorial collection.

[10] E. Fouvry and C. Mauduit, Méthodes de crible et fonctions sommes des chiffres, Acta Arith. 77(4) (1996), 339-351.

[11] E. Fouvry and C. Mauduit, Sommes des chiffres et nombres presque premiers, Math. Ann. 305(3) (1996), 571-599.

[12] C. Frougny. Representations of numbers and finite automata, Math. Systems Theory 25(1) (1992), $37-60$.

[13] A. O. Gel'fond, Sur les nombres qui ont des propriétés additives et multiplicatives données, Acta Arith. 13 (1967/1968), 259-265.

[14] P. J. Grabner, R. F. Tichy, I. Nemes, and A. Pethő, Generalized Zeckendorf expansions, Appl. Math. Lett. 7(2) (1994), 25-28.

[15] P. J. Grabner, R. F. Tichy, I. Nemes, and A. Pethö, On the least significant digit of Zeckendorf expansions, Fibonacci Quart. 34(2) (1996), 147-151. 
[16] P. Kirschenhofer, H. Prodinger, and R. F. Tichy, Über die Ziffernsumme natürlicher Zahlen und verwandte Probleme. In Zahlentheoretische Analysis, volume 1114 of Lecture Notes in Math. (Springer, Berlin, 1985), pp. 55-65.

[17] C. Mauduit, C. Pomerance, and A. Sárközy, On the distribution in residue classes of integers with a fixed sum of digits, Ramanujan J. 9(1-2) (2005), 45-62.

[18] C. Mauduit and A. Sárközy, On the arithmetic structure of sets characterized by sum of digits properties, J. Number Theory 61(1) (1996), 25-38.

[19] C. Mauduit and A. Sárközy, On the arithmetic structure of the integers whose sum of digits is fixed. Acta Arith. 81(2) (1997), 145-173.

[20] W. Parry, On the $\beta$-expansions of real numbers, Acta Math. Acad. Sci. Hungar. 11 (1960), 401-416.

[21] A. Pethő and R. F. Tichy, On digit expansions with respect to linear recurrences, J. Number Theory 33(2) (1989), 243-256.

[22] E. Zeckendorf, Représentation des nombres naturels par une somme de nombres de Fibonacci ou de nombres de Lucas, Bull. Soc. Roy. Sci. Liège 41 (1972), 179-182. 